\title{
A Novel NFC Antenna for Metal Cover Smartphone Applications
}

\author{
Jia-Qi Zhu, Yong-Ling Ban, Rui-Min Xu, and Ji-Wei Lian \\ School of Electronic Engineering \\ University of Electronic Science and Technology of China \\ Chengdu, Sichuan, China \\ byl@uestc.edu.cn
}

\begin{abstract}
A miniaturized dual-loop near field communication antenna of size $15 \mathrm{~mm} \times 25 \mathrm{~mm}$ is proposed for metal cover smartphone applications. By embedded it with a small rectangular slot of size $8 \mathrm{~mm} \times 16 \mathrm{~mm}$ loaded metal cover, the direction of the eddy current induced on metal cover will be the same with the current flow the on the dual loop NFC antenna coil. It's worth noting that the proposed NFC antenna design was certified by the Europay, Mastercard and Visa (EMV) test.
\end{abstract}

Keywords-miniaturized NFC antenna, full metal-cover, rectangular slot, dual-loop coil, EMV

\section{INTRODUCTION}

NFC is a near field technology working within a short range, with an operating frequency of $13.56 \mathrm{MHz}$ [1]. Nowadays, it has become an indispensable element embedded in commercial smartphones. Conventional NFC antenna designs for non-metallic metal cover smartphones usually apply ferrite composite to shield the reverse eddy current induced on metal mainboard and battery [2]. However, for metal cover smartphones, NFC antenna is sandwiched between metal cover and metal mainboard. Reverse eddy current induced on metal cover will reduce passive parameters such as self inductance $\left(L_{a}\right)$ and quality factor $(Q)$, thus leading to poor performance[3].

An NFC antenna working alongside the touch panel for Tablet PC with metal cover was reported in [4]. To avoid eddy current on metal cover, the NFC antenna was allocated beneath the large clearance zone of $41.5 \mathrm{~mm} \times 7.5 \mathrm{~mm}$. An NFC antenna with non-uniform meandering line and partial coverage ferrite sheet is reported in [5]. It only requires a main horizontal slot of $2 \mathrm{~mm}$ width. However, it also has the overlapping problem with other antenna applications because of the position and large planar size $(46 \mathrm{~mm} \times 38 \mathrm{~mm})$ of the coil.

In this paper, a novel miniaturized dual-loop NFC antenna $(15 \mathrm{~mm} \times 25 \mathrm{~mm})$ with a very small square slot clearance $(8$ $\mathrm{mm} \times 16 \mathrm{~mm}$ ) is proposed for achieving NFC in full metal cover smartphone applications. Besides the small dimensions of the antenna coil and clearance zone, it also overcomes the overlapping problem. Furthermore, it was certified by EMV test.

\section{NFC ANTENNA STRUCTURE AND Other COMPONENTS}

The structure and detailed dimensions of the proposed dualloop NFC antenna coil is shown in Fig. 1(a). The antenna coil

\author{
Chow-Yen-Desmond Sim \\ Department of Electrical Engineering \\ Feng Chia University \\ Taichung, Taiwan \\ cysime@fcu.edu.tw
}

is $15 \mathrm{~mm}$ in length and $25 \mathrm{~mm}$ in width. The coil consists of two loops, the 6 turn upper loop and 5 turn lower loop. They are connected by a jumper line. For better understanding, the NFC antenna is divided into five sections. The line width and line gap of the top and bottom section are $0.5 \mathrm{~mm}$ and $0.4 \mathrm{~mm}$, respectively. The corresponding left and right sections have shared the line width and gap of $0.4 \mathrm{~mm} 0.3 \mathrm{~mm}$, respectively. As for the middle section, the width and gap of it are $0.3 \mathrm{~mm}$ and $0.2 \mathrm{~mm}$, respectively. The breakdown diagram is so much similar as [5]. For brevity, it's not shown here.

The plan view of the proposed NFC antenna and other components are shown in Fig. 1(b). The antenna coil assembled with ferrite composite is sandwiched between metal cover (planer size of $41.5 \mathrm{~mm} \times 7.5 \mathrm{~mm}$ ) and metal mainboard (planer size of $64 \mathrm{~mm} \times 66.1 \mathrm{~mm}$ ). It's noteworthy that the NFC antenna is embedded directly beneath the center of the rectangular slot. Furthermore, the minor gap between the mainboard and upper edge of the metal cover is only $1.7 \mathrm{~mm}$.

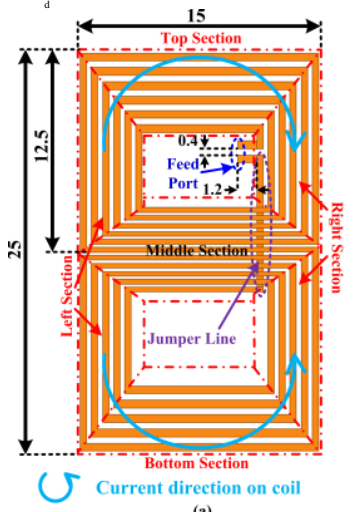

(a)

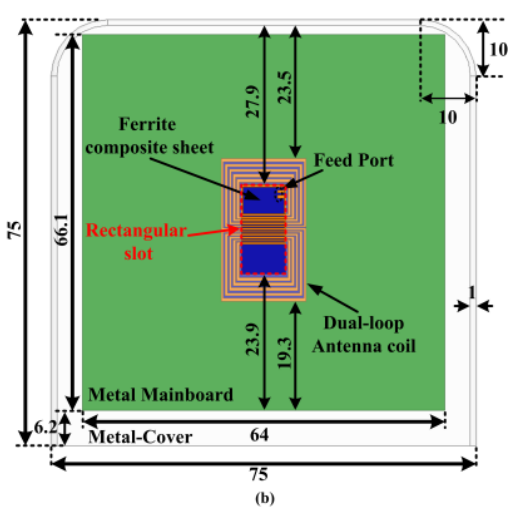

(b)
Fig. 1. (a) Structure and detailed dimensions of the proposed dual-loop NFC antenna coil, (b) plan view of the proposed dual-loop NFC antenna and other components. (Units: $\mathrm{mm}$ )

\section{NFC ANTENNA DESIGN}

Eddy current distributions induced on the inner and outer surfaces of metal cover are plotted in Fig. 2(a) and Fig. 2(b), respectively. It's worth noting that the same and reverse direction of the eddy current on metal cover are compared with the current direction on NFC antenna coil (blue color). As depicted in Fig. 2, within the inner surface of metal cover, only the eddy current induced directly above the NFC antenna coil is in reverse direction with the current flow (blue color) on the antenna coil. The eddy current exhibited on the remaining inner and outer surface area (red color) are all in the same 
direction compared with the current flow (blue color) on antenna coil. Since the majority of eddy current (red color) are in the same direction with the current flow on NFC antenna (blue color), good magnetic field distribution is expected. For comparison purpose, a 6 turn conventional reference antenna coil (planer size of $15 \mathrm{~mm} \times 25 \mathrm{~mm}$ ) is introduced in Fig. 3 . Here, the corresponding line width and line gap of the reference antenna coil are the same with the proposed NFC antenna. The magnetic field distribution plotted $10 \mathrm{~mm}$ away from the reference antenna coil and proposed dual-loop antenna coil are shown in Fig. 3(a) and (b), respectively. Note that the conditions between the two antennas are exactly the same, except that the two antennas are of different structure. Thus, compared with the reference antenna, huge improvement in the magnetic field of the proposed antenna shows the advantage of the proposed dual-loop NFC antenna.

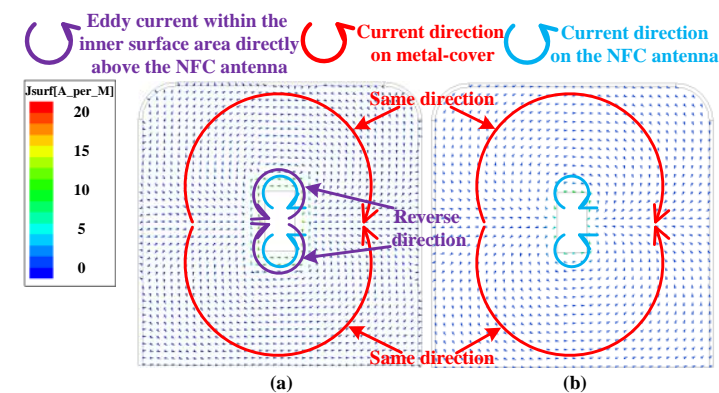

Fig. 2. (a) Eddy current distributions of the proposed NFC antenna on the inner surface of metal cover, (b) Eddy current distributions of the proposed NFC antenna on the outer surface of metal cover.
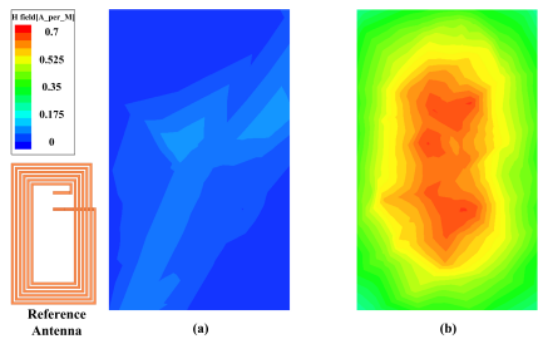

Fig. 3. Magnetic field strength distributions plotted $10 \mathrm{~mm}$ away from the (a) conventional antenna coil (reference antenna coil), (b) proposed antenna coil.

\section{Discussion AND MEASUREMENT}

Before showing the measurement results, it's important to discuss the impact of the proposed NFC antenna on other antennas. To support our argument that the proposed NFC antenna will not interfere with other antennas, except for very unusual cases when other antennas are stacking above or in very close proximity with our proposed NFC antenna, a simple reference inverted-F antenna (IFA) with switching capability is designed, as shown in Fig. 4. The return loss of this IFA with and without loading the proposed NFC antenna are almost identical with each other. Due to the two pages limitation, the results are not shown here.

The front view and back view of the fabricated proposed dual-loop NFC antenna and other components are shown in Fig. 5(a) and Fig. 5(b), respectively. It's noteworthy that the metal cover was practically constructed by gluing copper sheets on a plastic mould. After connected the NFC antenna to PN548 demo board, EMV test for card emulation (CE) mode was conducted. The results for EMV test are shown in Table I .

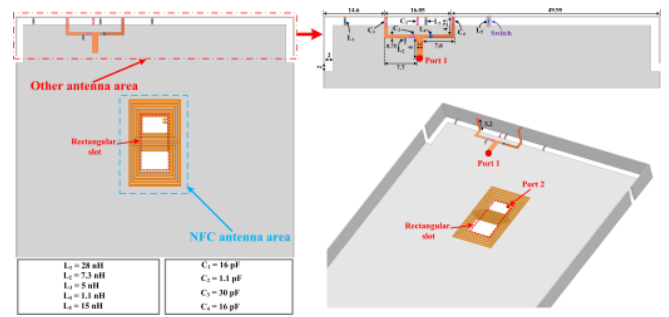

Fig. 4. The structure and detailed dimension of NFC antenna and the reference IFA with switching capability.

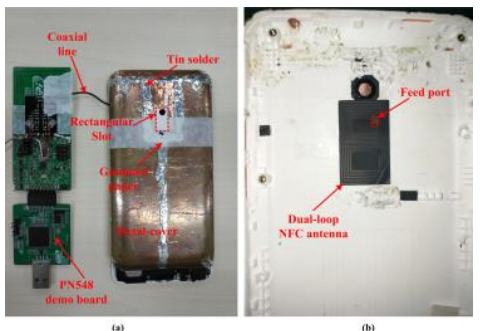

Fig. 5. The fabricated proposed dual-loop NFC antenna and other components, (a) front view, (b) back views.

TABLE I. MEASURED RESULTS FOR CARD EMULATION (CE) AND DETECTION RANGE OF THE R/W MODE

\begin{tabular}{|c|c|c|c|c|c|c|c|}
\hline $\mathbf{r}, \Phi$ & \multicolumn{7}{|c|}{ Load Modulation Level [mV $\mathrm{mPP}_{\mathrm{PP}}$} \\
\hline \multirow{3}{*}{$\begin{array}{c}\mathrm{Z} \\
(\mathrm{cm})\end{array}$} & \multicolumn{2}{|c|}{$\mathbf{r}=\mathbf{0}(\mathbf{c m})$} & \multicolumn{5}{|c|}{$r=1.5(\mathrm{~cm})$} \\
\hline & \multirow{2}{*}{$\begin{array}{c}\text { Min/ } \\
\text { Max. Spec. }\end{array}$} & \multirow{2}{*}{$\begin{array}{c}\Phi \\
0^{\circ}\end{array}$} & \multirow{2}{*}{$\begin{array}{c}\text { Min/ } \\
\text { Max. Spec. }\end{array}$} & \multicolumn{4}{|c|}{$\Phi$} \\
\hline & & & & $0^{\circ}$ & $90^{\circ}$ & $180^{\circ}$ & $270^{\circ}$ \\
\hline 0 & $8.8 / 80$ & 17.4 & $4.9 / 80$ & 16.8 & 6.1 & 14.8 & 43.6 \\
\hline 1 & $7.2 / 80$ & 8.8 & $4.1 / 80$ & 9.1 & 4.6 & 7.3 & 21.9 \\
\hline 2 & $5.6 / 80$ & 5.0 & $3.3 / 80$ & 5.3 & 3.5 & 4.2 & 9.7 \\
\hline 3 & $4.0 / 80$ & 4.1 & & & & & \\
\hline
\end{tabular}

The Detection Range for R/W Mode Test is $21 \mathrm{~mm}$.

\section{CONCLUSION}

In this paper, a miniaturized dual loop NFC antenna (15 $\mathrm{mm} \times 25 \mathrm{~mm})$ embedded with a rectangular slot $(8 \mathrm{~mm} \times 16$ $\mathrm{mm}$ ) loaded metal cover has been successfully conducted and studied. The proposed NFC antenna has achieved relatively good card emulation performance with detection range of 21 $\mathrm{mm}$ in $\mathrm{R} / \mathrm{W}$ mode. Furthermore, potential overlapping problem is eliminated. Thus, it can be applied in actual smartphones.

\section{REFERENCES}

[1] J. Fischer, "NFC in cell phones: the new paradigm for an interactive world," IEEE Communications Mag., vol. 47, no. 6, pp. 22-28, Jun 2009.

[2] B. Lee, B. Kim, F. J. Harackiewicz, B. Mun, and H. Lee, "NFC antenna design for low-permeability ferromagnetic material," IEEE Antennas Wireless Propag., vol. 13, pp. 59-62, 2014.

[3] X. Qing and Z. N. Chen, "Proximity effects of metallic environments on high frequency RFID reader antenna: study and applications," IEEE Trans. Antennas Propagat., vol. 55, no.11, pp. 3105-3111, Nov 2007.

[4] M. A. Chung and C. F, Yang, "Miniaturized NFC Antenna Design for a Tablet PC With a Narrow Border and Metal Back-Cover," IEEE Antennas Wireless Propag., vol. 15, pp. 1470-1474, Dec 2015.

[5] J. Q. Zhu, Y. L. Ban, and C. Y. D. Sim, "NFC Antenna with NonUniform Meandering Line and Partial Coverage Ferrite Sheet for Metal Cover Smartphone Applications," IEEE Trans. Antennas Propagat., vol. 65, no. 6, pp. 2827-2835, Jun 2017.

[6] EMV contactless specification for payment systems: Book D, EMV contactless communication protocol specification; Rev. 2.2. EMVco; 2012 . 